\title{
Análisis cuantitativo de la investigación en invasiones biológicas en Chile: tendencias y desafíos
}

\author{
Quantitative analysis of the research in biological invasions in Chile: \\ Trends and challenges
}

\author{
CONSTANZA L. QUIROZ ${ }^{1,2, ~ *}$, ANÍBAL PAUCHARD ${ }^{2,1}$, LOHENGRIN A. CAVIERES $^{1,3}$ \\ \& CHRISTOPHER B. ANDERSON ${ }^{1,4}$
}

\author{
${ }^{1}$ Instituto de Ecología y Biodiversidad (IEB), Casilla 653, Santiago, Chile \\ ${ }^{2}$ Facultad de Ciencias Forestales, Universidad de Concepción, Casilla 160-C, Concepción, Chile \\ ${ }^{3}$ ECOBIOSIS, Departamento de Botánica, Facultad de Ciencias Naturales y Oceanográficas, Universidad de Concepción, \\ Casilla 160-C, Concepción, Chile \\ ${ }^{4}$ Parque Etnobotánico Omora, Universidad de Magallanes, Puerto Williams, Chile \\ *Autor correspondiente: cquiroz@udec.cl
}

\begin{abstract}
RESUMEN
En las últimas dos décadas, el estudio de las invasiones biológicas ha alcanzado un auge sin precedentes a nivel mundial tanto en sus dimensiones teóricas como aplicadas. En Chile, las invasiones biológicas parecieran ser un tema relativamente nuevo. En este artículo se evalúa la tendencia en la investigación de invasiones biológicas en Chile entre los años 1991 y 2008. El análisis se realizó en plantas y animales, de acuerdo a las temáticas abarcadas en los estudios, y las aproximaciones metodológicas utilizadas. La serie temporal de trabajos publicados en invasiones biológicas en Chile está relacionada significativamente con la tendencia de publicaciones sobre invasiones biológicas a nivel mundial. La mayoría de los estudios en Chile se enfocaron en la etapa de invasión de especies introducidas, seguida por las etapas de naturalización de especies y finalmente de introducción. El estudio de patrones de invasión ha sido evaluado con mayor frecuencia en plantas introducidas que en animales, mientras que el estudio del impacto de especies introducidas muestra la tendencia contraria. La mayor parte de los estudios realizados tanto en plantas como animales introducidos se ejecutaron a partir de muestreos, seguidos por estudios experimentales. Concluimos que existen aún importantes desafíos para los investigadores enfocados en el tema de invasiones biológicas, incluyendo: 1) no solo buscar describir patrones sino también entender procesos detrás de estos patrones, 2) incorporar más estudios experimentales, especialmente enfocados a la evaluación de impactos, 3) mejorar la integración de los estudios a la conservación y restauración de ecosistemas, y 4) intentar conectar los estudios locales a los actuales marcos conceptuales.
\end{abstract}

Palabras clave: ecología de invasiones, especie adventicia, especie exótica, productividad científica.

\begin{abstract}
During the last decades, the study of biological invasions has reached an unforeseeable peak both in the theoretical and applied dimensions. In this article, we assess the trends in research on biological invasions in Chile between the years 1991 and 2008. The analysis was performed for animals and plants, according to the focus of the study, and the methods used in the article. The time series of published articles on biological invasions in Chile is significantly related to the number of publications on biological invasions world-wide. We found that most studies in Chile have focused on the invasion of introduced species, followed by the naturalization of species and finally, species introduction. The study of invasion patterns has more often been evaluated in introduced plants than animals, while the study of the impact of introduced species shows the opposite trend. Most studies of introduced plants and animals have used a sampling approach, followed by experimental studies. We conclude that there are still some important challenges for researchers focused on biological invasions, including: 1) along with describing patterns, the studies should also aim to understand the processes behind these patterns, 2) more experimental work should be incorporated, specially assessing invasion impacts, 3) a greater integration should exist between conservation and ecosystem restoration, and 4) local studies should consider current conceptual frameworks.
\end{abstract}

Key words: alien, exotics, invasion ecology, non-native, scientific productivity. 


\section{INTRODUCCIÓN}

Pese a que la introducción de especies más allá de su distribución nativa es un proceso generado por causas tanto naturales como antropogénicas desde hace milenios, la actual tasa de introducciones facilitadas por los seres humanos en asociación con la alteración de hábitat está generando una biota global cada vez más homogeneizada (McKinney \& Lockwood 1999). Además, muchas de estas especies introducidas invaden comunidades nativas, disminuyendo su diversidad y alterando sus servicios ecosistémicos (Mack et al. 2000). Por lo tanto, las invasiones biológicas resultan de interés científico tanto por su efecto en los ecosistemas y la biodiversidad como también por ser consideradas uno de los experimentos naturales más atractivos en biogeografía y ecología (Callaway \& Maron 2006).

En las últimas dos décadas, el estudio de la ecología de las invasiones biológicas ha alcanzado un auge sin precedentes tanto en sus dimensiones teóricas como aplicadas (Lockwood et al. 2007). "The Ecology of Invasions by Animals and Plants", realizada a fines de los años 50 (Elton 1958), es reconocida como la publicación fundadora del tema, aunque el interés por estudiar el comportamiento de especies introducidas se remonta a lo menos hasta Darwin (Darwin 1859). Sin embargo, el estudio de las invasiones biológicas no atrajo especialmente el interés de los ecólogos sino hasta fines de los años 70 (Davis 2006), período en que se comenzó a reconocer por parte de la comunidad científica, y la sociedad en general, del aumento en la tasa, escala y magnitud del efecto antrópico sobre el planeta (Vitousek et al. $1997 \mathrm{a}, \mathrm{b})$.

El estudio de la ecología de las invasiones biológicas, como subdisciplina de la ecología, se consolidó solo en los años 80, con un número creciente de artículos sobre invasiones durante esa década y la publicación del libro "Biological Invasions: A Global Perspective" (Drake et al. 1989). A partir de los años 90, el interés por las invasiones biológicas se instaló también en la toma de decisiones ambientales. Es así que el uso del término invasiones biológicas llegó a constituir no solo un marco conceptual para la investigación en ecología, sino también una metáfora para la comunicación a la sociedad. En este sentido, el concepto ha sido útil para plantear el tema ante los tomadores de decisiones sobre manejo de recursos naturales, posicionándolo en un rol de mayor protagonismo en las agendas ambientales globales. La importancia que ha cobrado el tema queda reflejada en las iniciativas internacionales dedicadas exclusivamente a este tema a nivel mundial. Por ejemplo, el "Programa Global de Especies Invasoras" (GISP), tiene por objetivo el seguimiento de especies invasoras a nivel global, mientras que el "Grupo de Especialistas en Especies Invasoras" (ISSG), tiene por misión aconsejar respecto al tema de invasiones biológicas a organismos gubernamentales y organismos dedicados a la conservación.

En Chile, la investigación bajo el marco conceptual de las invasiones biológicas solo se ha desarrollado en los últimos años (Pauchard et al. 2004). Las revisiones de Jaksic (1998), en vertebrados terrestres, y Arroyo et al. (2000), en plantas vasculares, pueden ser considerados como trabajos seminales de un desarrollo sistemático de la ecología de invasiones en Chile. Si bien, antes del año 2000, existía investigación sobre especies introducidas, esta no se realizó bajo el marco conceptual de las invasiones biológicas. Por ejemplo, Reiche (1903) reporta varias especies introducidas en Chile y describe cómo identificarlas para eliminarlas de los cultivos donde actúan como malezas. Otros autores como Baeza (1928) y Behn (1945), regularmente publicaban artículos donde daban a conocer el hallazgo de especies no nativas de Chile simplemente con el ánimo de identificarlas. Por lo tanto, parece razonable preguntarse cuánto hemos avanzado en Chile respecto el estudio de las invasiones biológicas y qué nos queda por hacer.

En este artículo evaluamos la tendencia en la investigación sobre invasiones biológicas en Chile, utilizando como indicador las publicaciones generadas en el tema bajo este marco conceptual. Analizando estas publicaciones, identificamos las principales temáticas abordadas hasta ahora tanto en plantas como en animales, así como aquellas donde se ha avanzado menos. A partir de esto último, presentamos una propuesta de priorización para futuros estudios con el fin de avanzar con mayor celeridad en algunos ámbitos, especialmente en aquellos más estrechamente relacionados con los 
aspectos aplicados del estudio de la ecología de invasiones.

\section{Analizando el estudio de invasiones biológicas en Chile}

Realizamos una búsqueda bibliográfica de artículos publicados utilizando las palabras clave "Chile" + "invasión" o "Chile" + "exotic" en los buscadores "ISI Web of Knowledge" y SciELO. Además, realizamos una búsqueda específica en los sitios web de los principales centros de investigación en invasiones biológicas en Chile, como el Instituto de Ecología y Biodiversidad (IEB), y el Centro de Estudios Avanzados en Ecología y Biodiversidad (CASEB). En esta búsqueda se incluyó tanto revistas indexadas como no indexadas, y capítulos de libros. El listado final de artículos incluyó también las publicaciones del tipo revisión y publicaciones que se encontraban en prensa al momento de la búsqueda, a las que logramos tener acceso (e.g. Pauchard et al. 2009).

Para contrastar el desarrollo de la subdisciplina, comparamos el total de las publicaciones anuales generadas en invasiones biológicas en Chile con los números totales de publicaciones sobre ecología (sensu lato) en Chile y sobre invasiones biológicas a nivel mundial de acuerdo a los datos del ISI Web of Knowledge para cada año. El número de publicaciones sobre ecología en Chile se obtuvo a partir de una búsqueda de todas las publicaciones registradas bajo los filtros "Topic: Chile" + "Refine by: Ecology". El número de publicaciones sobre invasiones biológicas a nivel mundial se obtuvo a partir de una búsqueda bajo el filtro "Topic: invasi* OR alien* OR exotic*" + "Refine: Ecology". El periodo en que se centraron estas últimas dos búsquedas fue ajustado de acuerdo al periodo en que fueron publicados los artículos sobre invasiones biológicas en Chile. Para evaluar cuál de las dos series temporales predice mejor el número de trabajos publicados en invasiones biológicas en Chile a través del tiempo, realizamos un análisis de regresión múltiple. En este análisis, se examinó la importancia relativa de cada una de las series temporales consideradas como variables predictoras, i.e. número de publicaciones en ecología general en Chile, y número de publicaciones sobre invasiones biológicas a nivel mundial.

Se revisó cada uno de los artículos encontrados sobre invasiones biológicas en Chile y para cada uno se estableció: (a) la(s) etapa(s) del proceso de invasión de la especie introducida (introducción, naturalización, invasión) y (b) el taxón estudiado, animal o planta. La definición utilizada para catalogar las etapas "introducción", y "naturalización" fue la propuesta por Richardson et al. (2000), mientras que la de "invasión" fue modificada según Dietz \& Edwards (2006). Por etapa "invasión" entendemos una especie naturalizada (según Richardson et al. 2000), que es capaz de mantenerse sin ayuda humana en comunidades seminaturales ("invasión secundaria" sensu Dietz \& Edwards 2006). Los trabajos publicados se clasificaron en cinco criterios de acuerdo a la temática estudiada (modificado de Pauchard et al. 2004): desarrollo de inventarios de especies introducidas, indicando la presencia/ausencia de estas (Pre), documentación de los patrones de invasiones en comunidades nativas (Pat), estudio de los procesos de invasiones en comunidades nativas de Chile (Pro), estudio de la autoecología de la especie introducida (Aut), evaluación de impactos económicos y ecológicos de la especie introducida (Imp), u otros temas tratados (Otros). Posteriormente, la aproximación utilizada para obtener los datos fue dividida en experimental, herbario, muestreo, revisión y simulación. Debido a que varios artículos evalúan más de una etapa del proceso de invasión, más de una temática y/o utilizan más de una aproximación para obtener los datos, los porcentajes totales para cada una de las clasificaciones podrían sumar más de 100 $\%$. Las categorías no son excluyentes entre sí.

Tendencias encontradas en el estudio de las invasiones biológicas en Chile

La búsqueda de publicaciones sobre invasiones biológicas en Chile arrojó un total de 92 artículos, publicados entre enero de 1991 y diciembre de 2008 (Tabla C1, Material Complementario; http://rchn.biologiachile.cl/ suppmat/Quiroz_et_al_2009_MC.pdf). Si bien existen estudios en especies introducidas previos a este periodo en Chile, este marco temporal está determinado por el acceso a la información disponible en Internet. La cantidad de artículos 
publicados ha crecido principalmente a partir del año 2003 (Fig. 1), observándose un mayor porcentaje en los últimos cuatro años (20052008) respecto a la cantidad total de artículos encontrados en esta búsqueda (1991-2008).

Entre las dos variables independientes postuladas para explicar la variación temporal en el estudio de invasiones biológicas en Chile, solo la tendencia internacional de publicaciones sobre invasiones biológicas fue seleccionada como variable significativa $\left(F_{1,15}=17.33 ; P=0.0008\right)$. La tendencia de publicaciones en ecología en Chile no resultó ser una variable predictora significativa en el análisis $\left(\mathrm{F}_{1,15}=0.027 ; \mathrm{P}=0.8717\right)$. En Chile, la publicación de estudios en ecología ha sido más constante que la publicación de artículos sobre invasiones biológicas, lo que explicaría la exclusión de esta variable para predecir la tendencia en el número de publicaciones sobre invasiones biológicas en Chile.

Al examinar el total de los estudios sobre invasiones biológicas en Chile, la mayoría se han enfocado en la etapa de invasión de especies introducidas $(63 \%)$, seguida por la naturalización de especies $(51 \%)$ y finalmente la introducción de especies (12\%) (Fig. 2). Por otro lado, la mayor parte de los artículos analizados estuvieron enfocados en especies de plantas $(59 \%)$ mientras que el $46 \%$ de los artículos incluyen especies animales introducidas. El $7 \%$ de las publicaciones en invasiones biológicas en Chile involucran tanto animales como plantas introducidas.

La mayor parte de los estudios en plantas se enfocan en el estudio de los patrones de especies invasoras en comunidades naturales (33\%), mientras que el resto de los criterios tuvo una variada representación en los estudios. De hecho, el $83 \%$ de los estudios enfocados en especies de plantas abarcan al menos uno de los criterios seleccionados (Fig. 3), aunque la mayoría abarca más de uno a la vez. Por ejemplo, Neill et al. (2006) analizó los patrones de la invasión de Codium fragile spp. tomentosoides (van Goor) Silva en la costa del norte de Chile, además de enfocarse en la autoecología de la especie involucrada y el impacto que esta genera.

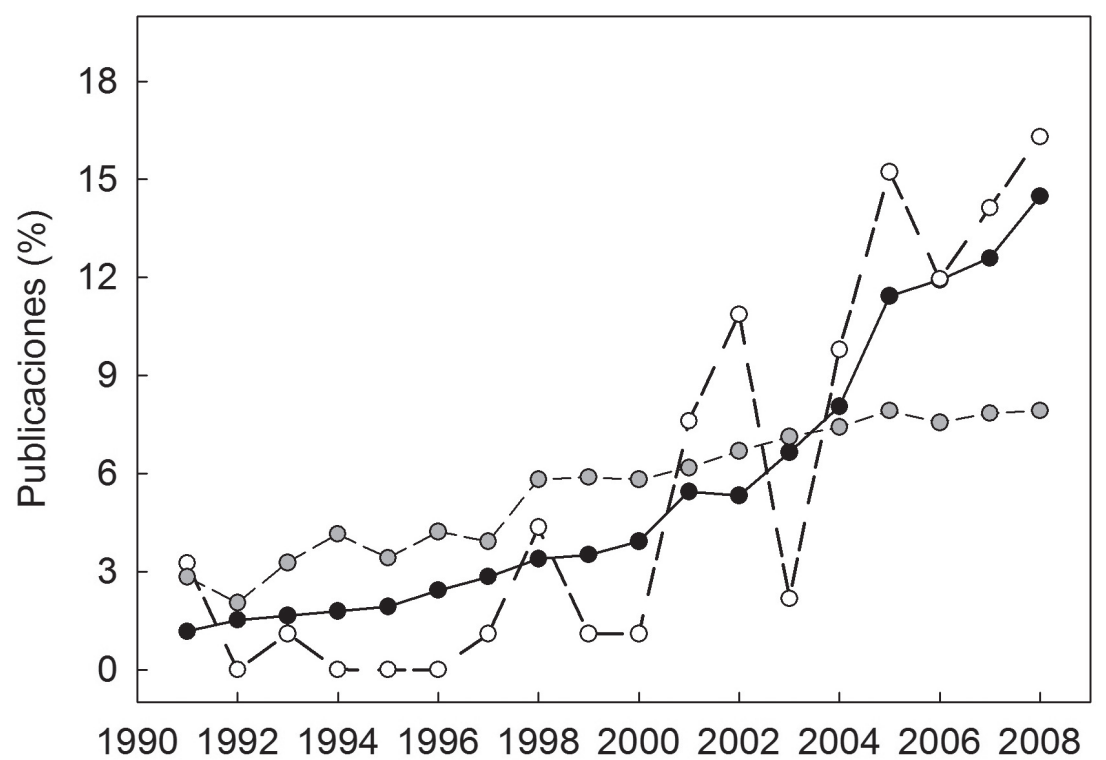

Fig. 1: Porcentaje de publicaciones generadas cada año en el periodo 1991-2008. Los porcentajes para cada año fueron calculados a partir del total de artículos encontrados en el periodo 1991-2008. Los círculos blancos representan las publicaciones generadas en Chile en torno al tema de invasiones biológicas (tanto ISI como no ISI). Los círculos grises representan las publicaciones ISI generadas en Ecología en Chile. Los círculos negros representan las publicaciones ISI generadas en Invasiones Biológicas a nivel mundial.

Percentage of publications generated each year during the period 1991-2008. The percentages for each year were calculated from the total number of articles found in the period 1991-2008. White circles represent the articles generated in Chile about biological invasions (both ISI and non-ISI). Grey circles represent the ISI publications generated in ecology in Chile. Black circles represent ISI publications generated about biological invasions in the world. 


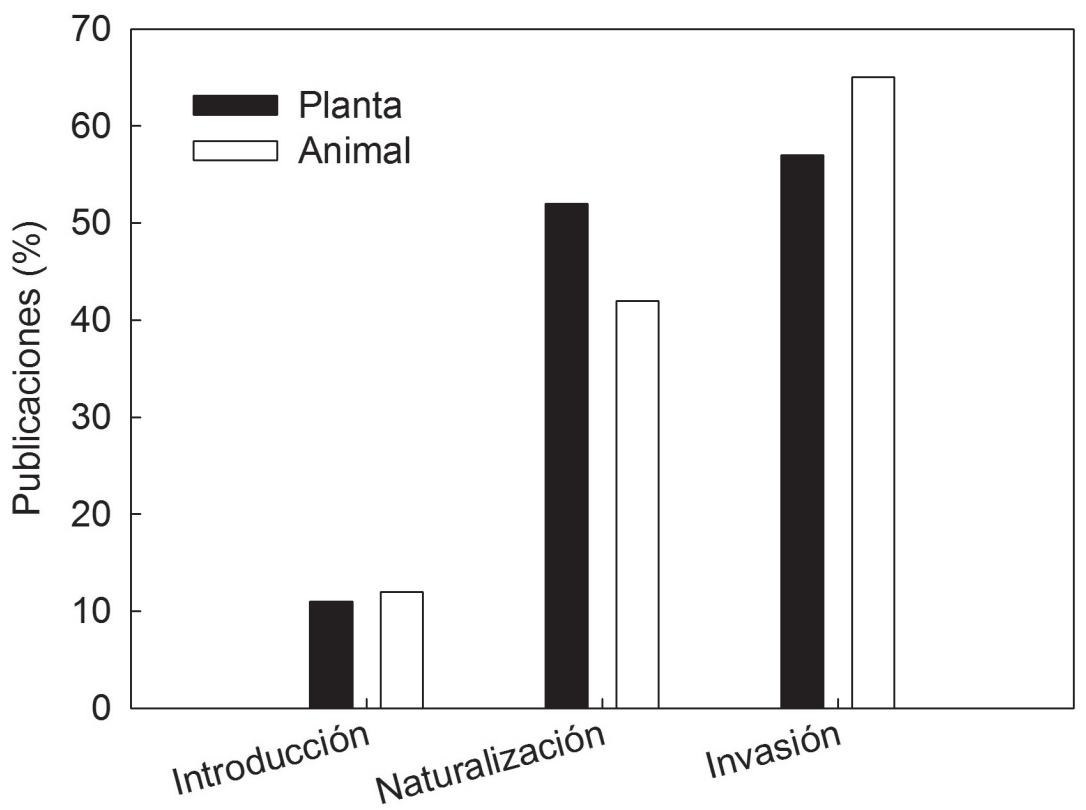

Fig. 2: Etapa del taxón introducido evaluada en las publicaciones incluidas en el estudio, separadas por el tipo de taxón examinado (animal o planta). La definición utilizada para catalogar las etapas introducción, y naturalización fue la propuesta por Richardson et al. (2000), mientras que la de invasión fue modificada según Dietz \& Edwards (2006). Por etapa invasión entendemos una especie naturalizada (según Richardson et al. 2000), que es capaz de mantenerse sin ayuda humana en comunidades seminaturales ("invasión secundaria" sensu Dietz \& Edwards 2006). El número de publicaciones es representado como el porcentaje de publicaciones respecto al total de publicaciones incluidas en el estudio. Si un artículo abarca más de una etapa, o ambos taxa, este fue registrado más de una vez en el gráfico.

Stage of the introduced taxon evaluated in the publications included in this study, separated by the type of examined taxon (animal or plant). The definition used for separating the stages of introduction and naturalization was proposed by Richardson et al. (2000), while the invasion stage was modified according to Dietz \& Edwards (2006). By invasion stage we understand a naturalized species (according to Richardson et al. 2000), that is able to maintain self-perpetuating populations in semi-natural communities ("secondary invasion" sensu Dietz \& Edwards 2006). The number of publications is represented as the percentage of publications from the total number of publications included in this study. If an article examines more than one stage, or both taxa, it was registered more than once in the graph.

Los estudios que abarcan el desarrollo de inventarios de especies introducidas han utilizado principalmente datos obtenidos a partir de muestreos realizados en terreno y revisión de datos publicados, al igual que los patrones de invasión en comunidades nativas (Fig. 3). Por ejemplo, Castro et al. (2005) utilizaron datos obtenidos de revisiones bibliográficas y de herbario para evaluar los determinantes del avance geográfico de plantas naturalizadas en Chile. Los procesos por los cuales se da la invasión de comunidades nativas han sido estudiados a través de aproximaciones experimentales, aunque también se han utilizado datos obtenidos a través del muestreo de comunidades naturales (Fig. 3) (e.g., Badano et al. 2007). Múltiples aproximaciones han sido utilizadas para estudiar la autoecología de especies introducidas, entre las que destacan la revisión de datos previamente publicados, muestreo y experimentos (Fig. 3).

Paradójicamente, a pesar de la preocupación que generan las invasiones biológicas, el impacto ecológico de especies de plantas introducidas ha sido escasamente evaluado (15 $\%)$. Los pocos estudios que evalúan el efecto de la presencia de estas especies en comunidades nativas han privilegiado la revisión de datos publicados. Por ejemplo, Castilla et al. (2005) presentan una revisión de impactos documentados producidos por especies de algas introducidas. Estos autores muestran impactos negativos de la presencia de especies introducidas sobre cultivos de algas nativas, 
aunque también reportan impactos positivos en la biodiversidad, generados a través de la creación de nuevos ambientes para la biodiversidad nativa por especies introducidas.

A pesar de no haber demarcado la búsqueda a ningún tipo de ser vivo, los estudios sobre animales que arrojó la búsqueda se circunscribieron principalmente a vertebrados. Las tendencias generales observadas en las publicaciones sobre animales introducidos respecto a las aproximaciones de estudio utilizadas y las etapas de invasión abordadas son similares a las descritas previamente para las plantas. Por ejemplo, la mayor parte de los estudios de animales introducidos se realizaron a partir de muestreos $(52 \%)$ y durante la etapa de naturalización $(67 \%)$. Al igual que en el caso de las plantas, las revisiones bibliográficas fueron importantes, siendo la segunda categoría más utilizada para la obtención de datos (38\%),

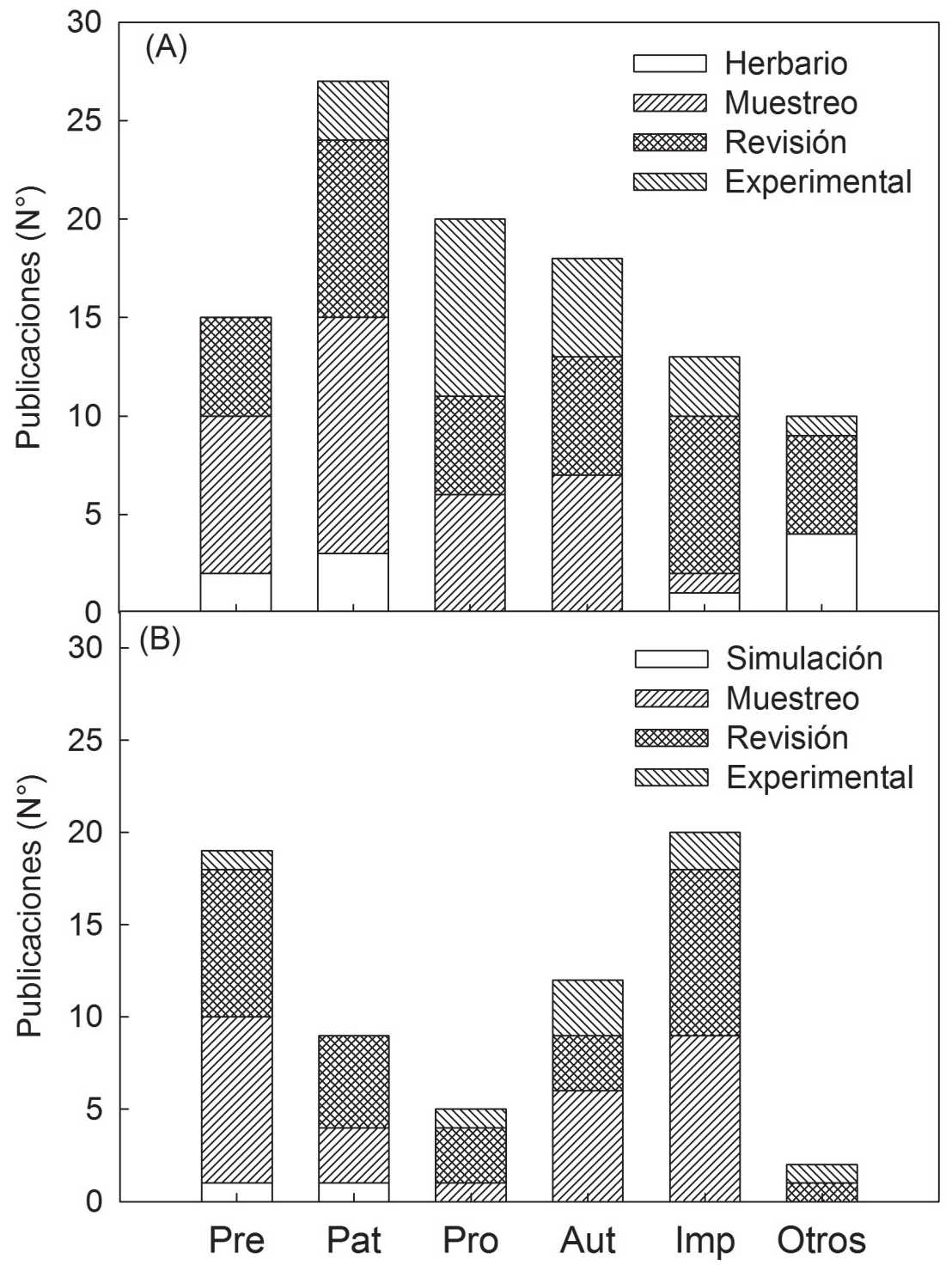

Fig. 3: Objetivo abarcado por publicaciones centradas en especies de plantas (A) o animales (B) introducidos, modificado a partir de Pauchard et al. (2004) (ver texto). Las publicaciones se encuentran subclasificadas por la aproximación utilizada para obtener los datos. Si un artículo abarca más de un objetivo, o más de una aproximación, este fue registrado más de una vez en el gráfico.

Objective used by publications centered in introduced plant species (A) or animal species (B), modified from Pauchard et al. (2004) (see text). Publications are sub-classified by the approach used for obtaining the data. If an article uses more than one objective, or more than one approach, it was registered more than once in the graph. 
superando la representatividad de la aproximación experimental (12\%).

Como en las plantas, los objetivos de los estudios de animales introducidos suelen ser la confección de inventarios, reportando la presencia/ausencia de especies $(43 \%)$. Sin embargo, una diferencia importante entre el estudio de las plantas y los animales fue que la cuantificación de impactos fue la categoría con mayor representatividad en la literatura de invasiones biológicas en Chile (45\%). Por su parte, la autoecología y la descripción de patrones de distribución fueron partes de los objetivos de $26 \%$ y $19 \%$ de los estudios respectivamente, mientras la explicación de los procesos (o mecanismos) sigue siendo el menos utilizado en los estudio de las invasiones de taxones animales con solo $12 \%$ de las investigaciones. Es probable que este sesgo en contra de explicaciones de mecanismos esté vinculado con alguna falta de interés por realizar estudios con manipulaciones experimentales.

Mientras a nivel mundial existe gran interés y esfuerzos significativos para no solo implementar programas de control y erradicación de especies invasoras, sino también generar investigación científica sobre el tema (e.g. Proyecto Isabela en las Islas Galápagos), se encontró una carencia de artículos cuya investigación haya sido desarrollada pensando explícitamente en políticas públicas sobre especies invasoras (sin embargo, ver Pérez et al. 2003), mientras comentarios y revisiones generales con implicancias para la toma de decisiones son más frecuentes.

Finalmente, evaluamos cuánta investigación realizada sobre invasiones biológicas en Chile se encuentra relacionada con la literatura internacional y con los marcos conceptuales actuales sobre el proceso de invasión. Entre los estudios evaluados, solo 23 artículos (25\%) encontrados han relacionado explícitamente (en el título o en el resumen) su estudio con teorías planteadas en la literatura internacional. Esta proporción es muy baja, considerando el gran beneficio que significaría para la disciplina en Chile apoyarse en marcos conceptuales desarrollados por grupos con mayor experiencia en el tema. Sin embargo, tampoco podemos descartar que el vínculo con la literatura internacional se encuentre en otras secciones del artículo, como por ejemplo la introducción o la discusión.

\section{CONCLUSIONES Y DESAFÍOS}

A pesar del gran aumento que el estudio de invasiones biológicas ha tenido en Chile en los últimos años, existen importantes desafíos pendientes.

Un número importante de los estudios (24 $\%)$ se restringen a descripciones de los patrones que siguen las especies introducidas. Sin embargo, para mejorar la comprensión y el control del proceso de invasión parece necesario ahondar en los mecanismos que lo permiten y, en algunos casos, lo restringen. Por ejemplo, Becerra \& Bustamante (2008) mostraron recientemente que el establecimiento de plántulas de dos especies invasoras es altamente restringido por la herbivoría de vertebrados, lo que podría impedir el avance de estas especies en ambientes donde la presión por herbívoros es mayor. Estudios como este abren nuevas posibilidades de control de especies invasoras, al identificar los mecanismos que restringen su avance.

Las investigaciones sobre el impacto de las especies introducidas son aún escasas en Chile. En este aspecto, sería beneficioso la incorporación de más estudios experimentales (véase Jaksic \& Fuentes 1991 para un estudio temprano y también Castilla et al. 2005), con el fin de adelantarse a las invasiones que pudieran ser más dañinas para la biodiversidad. Por ejemplo, Muñoz \& Cavieres (2008) manipularon la densidad de la especie naturalizada Taraxacum officinale Weber ex Wigg. en los Andes de Chile central y demostraron que esta provoca una disminución en la producción de semillas de especies nativas solo cuando presenta altas densidades poblacionales.

Los estudios sobre invasiones biológicas debieran tener una mayor orientación hacia las necesidades inmediatas de información para la toma decisiones en conservación. Para ello, es fundamental que el análisis de especies introducidas e invasoras esté estrechamente relacionado con programas de investigación que involucren no solo el estudio de los ecosistemas nativos sino también su restauración y el impacto social que pueda tener la especie de referencia. Dentro de los artículos revisados, solo uno de los estudios encontrados (Perez et al. 2003) se encuentra directamente dirigido hacia las entidades 
encargadas de lidiar con el problema de las invasiones biológicas, lo que muestra la urgencia de aumentar este tipo de estudios para aportar a la discusión de este tema en instancias de toma de decisiones. Debido a la amplia difusión que tienen las revistas científicas, la publicación de artículos sobre este tema podría abrir nuevas posibilidades de interacción con entidades relacionadas con las invasiones biológicas.

Finalmente, el estudio de invasiones biológicas tendrá un efecto relevante en ciencia y una aplicación satisfactoria solo si se logra encontrar las generalidades de los estudios locales. Para ello, es necesaria una mejor conexión de los estudios nacionales con la literatura internacional y con los marcos conceptuales que contribuyan al desarrollo teórico y aplicado.

Teniendo en cuenta las tendencias nacionales y globales en el estudio de invasiones biológicas, resulta evidente que la investigación en invasiones biológicas en Chile no es un fenómeno pasajero. Aún más, su relevancia debiera aumentar debido a sus implicancias en el fenómeno de cambio global y a la pérdida de biodiversidad (Sala et al. 2000). Cincuenta años después de la publicación del libro de Elton (1958), el interés en las invasiones biológicas es mayor que nunca, particularmente respecto a la conservación y manejo de especies introducidas, tanto dentro como fuera de la comunidad científica (Davis 2006).

Nuestra revisión del estado de la investigación sobre invasiones biológicas en Chile demuestra que, a pesar de que la investigación sobre invasiones biológicas lleva poco tiempo desarrollándose en Chile bajo un marco conceptual consolidado, la distribución de aproximaciones en la literatura es bastante equitativa, tanto entre los taxa evaluados, como en las etapas y preguntas realizadas. Un aumento en la investigación colaborativa aportaría a la integración de diferentes puntos de vista, aportando al desarrollo de la subdisciplina en nuestro país.

\section{AGRADECIMIENTOS}

Se agradece a Alejandro Valenzuela por una revisión preliminar del trabajo. A Sergio Castro por sus valiosas críticas y comentarios. Los autores son parcialmente financiados por el Laboratorio de Invasiones Biológicas (Proyectos ICM 05-002 y PFB-23 del Instituto de Ecología y Biodiversidad). AP y LC agradecen a Fondecyt 1070488.

\section{LITERATURA CITADA}

ARROYO MTK, C MARTICORENA, O MATTHEI \& LA CAVIERES (2000) Plant invasions in Chile: Present patterns and future predictions. En: Mooney $\mathrm{HA}$ \& RJ Hobbs (eds) Invasive species in a changing world: 385-421. Island Press, Washington, DC.

BADANO EI, E VILLARROEL, RO BUSTAMANTE, PA MARQUET \& LA CAVIERES (2007) Ecosystem engineering facilitates invasions by exotic plants in high-Andean ecosystems. Journal of Ecology 95: $682-688$

BAEZA VM (1928) Contribución al conocimiento de la flora advena de Chile: apuntes docentes. Instituto Pedagógico, Universidad de Chile, Santiago, Chile. 33 pp.

BECERRA PI \& RO BUSTAMANTE (2008) The effect of herbivory on seedling survival of the invasive exotic species Pinus radiata and Eucalyptus globulus in a Mediterranean ecosystem of Central Chile. Forest Ecology and Management 256: 1573-1578.

BEHN K (1946) Flora advena de Chile. Revista Universitaria (Chile) 31: 17-19.

CALLAWAY RM \& JL MARON (2006) What have exotic invasions taught us over the past twenty years? Trends in Ecology and Evolution 21: 369-374.

CASTILLA JC, M URIBE, N BAHAMONDE, M CLARKE, R DESQUEYROUX-FAÚNDEZ et al. (2005) Down under the southeastern Pacific: Marine non-indigenous species in Chile. Biological Invasions 7: 213-232.

CASTRO SA, JA FIGUEROA, M MUÑOZ-SCHICK \& FM JAKSIC (2005) Minimum residence time, biogeographical origin, and life cycle as determinants of the geographical extent of naturalized plants in continental Chile. Diversity and Distributions 11: 183-191.

DARWIN C (1859) The origin of species. J. Murray, London. 479 pp.

DAVIS MA (2006) Invasion biology 1958-2005: The pursuit of science and conservation. En: Cadotte MW, SM McMahon \& T Fukami (eds) Conceptual ecology and invasion biology: reciprocal approaches to nature: 35-64. Springer, Dordrecht, The Netherlands.

DIETZ H \& PJ EDWARDS (2006) Recognition that causal processes change during plant invasion helps explain conflicts in evidence. Ecology 87: 1359. 1367.

DRAKE JA, HA MOONEY, F DI CASTRI, RH GROVES, FJ KRUGER, M REJMANEK \& M WILLIAMSON (1989) Biological invasions: A global perspective. SCOPE 37, John Wiley \& Sons, Chichester, UK. $525 \mathrm{pp}$

ELTON CS (1958) The ecology of invasions by animals and plants. Methuen, London. $196 \mathrm{pp}$.

HOLMGREN M (2002) Exotic herbivores as drivers of plant invasion and switch to ecosystem alternative states. Biological Invasions 4: 25-33. 
JAKSIC FM (1998) Vertebrate invaders and their ecological impacts in Chile. Biodiversity and Conservation 7: 1427-1445.

JAKSIC FM \& ER FUENTES (1980) Why are native herbs in the Chilean matorral more abundant beneath bushes: Microclimate or grazing. Journal of Ecology 68: 665-669.

JAKSIC FM \& ER FUENTES (1991) Ecology of a successful invader: The European rabbit in central Chile. En: Groves RH \& F Di Castri (eds) Biogeography of Mediterranean Invasions: 273 284. Cambridge University Press, Cambridge, England.

McKINNEY ML \& JL LOCKWOOD (1999) Biotic homogenization: A few winners replacing many losers in the next mass extinction. Trends in Ecology and Evolution 14: 450-453.

LOCKWOOD JL, MF HOOPES \& MP MARCHETTI (2007) Invasion ecology. Blackwell Publishers. Malden, MA. 304 pp.

MACK RN, D SIMBERLOFF, WM LONSDALE, H EVANS, M CLOUT \& F BAZZAZ (2000) Biotic invasions: Causes, epidemiology, global consequences and control. Issues in Ecology 5: 1-25

MUÑOZ AA \& LA CAVIERES (2008) The presence of a showy invasive plant disrupts pollinator service and reproductive output in native alpine species only at high densities. Journal of Ecology 96: 459-467.

NEILL PE, O ALCALDE, S FAUGERON, SA NAVARRETE \& JA CORREA (2006) Invasion of Codium fragile ssp. tomentosoides in northern Chile: A new threat for Gracilaria farming. Aquaculture 259: 202-210.
PÉREZ JE, C ALFONSI, M NIRCHIO, C MUÑOZ \& JA GÓMEZ (2003) The introduction of exotic species in aquaculture: a solution or part of the problem? Interciencia 28: 234-238.

PAUCHARD A, LA CAVIERES, R BUSTAMANTE, P BECERRA \& E RAPPOPORT (2004) Increasing the understanding of plant invasions in southern South America: First symposium on Alien Plant Invasions in Chile. Biological Invasions 6: 255-257.

PAUCHARD A, C KUEFFER, H DIETZ, CC DAEHLER, J ALEXANDER et al. (2009) Ain't no mountain high enough: Plant invasions reaching new elevations. Frontiers in Ecology and the Environment 7: 479-486.

REICHE C (1903) Las malezas que invaden a los cultivos de Chile y el reconocimiento de sus semillas. Imprenta Franco-Chilena, Santiago, Chile. 88 pp.

RICHARDSON DM, P PYSEK, M REJMÁNEK, MG BARBOUR, FD PANETTA \& CJ WEST (2000) Naturalization and invasion of alien plants: Concepts and definitions. Diversity and Distribution 6: 93-107.

SALA OE, FS CHAPIN, JJ ARMESTO, E BERLOW, J BLOOMFIELD et al. (2000) Global biodiversity scenarios for the year 2100. Science 287: 17701774.

VITOUSEK PM, HA MOONEY, J LUBCHENCO \& J MELILLO (1997a) Human domination of Earth's ecosystem. Science 277: 494-499.

VITOUSEK PM, HA MOONEY, J LUBCHENCO \& JM MELILLO (1997b) Introduced species: A significant component of human-caused global change. New Zealand Journal of Ecology 21: 1-16. 
